\title{
Estrategias metodológicas para el aprendizaje significativo de la Química: estudio realizado en FAREM-Estelí, UNAN-Managua, 2016
}

\author{
Ana Teodora Téllez Flores ${ }^{1}$
}

\section{RESUMEN}

El presente artículo resume los resultados del estudio centrado en las estrategias metodológicas que propician aprendizajes significativos en Química, en dos carreras de la FAREM-Estelí, UNAN Managua, que se denominaron carrera número uno y carrera número dos. Se encontró que la conducción del proceso de aprendizaje en uno de los grupos clase fue adecuada y en el otro se presentaron algunos inconvenientes metodológicos y organizativos. Se comprobó que las estrategias metodológicas activas son las que las que propician aprendizajes significativos y motivación por estudiar la Química. Con este fin, se diseñó un compendió de estrategias metodológicas participativas, las que fueron adaptadas al contexto de nuestra Facultad como un apoyo a los docentes de Química y un aporte al proceso educativo. Estos resultados confirman nuestras intuiciones investigativas referidas a que, la utilización de estrategias metodológicas no activas limita el aprendizaje significativo Química, lo que está relacionado con la falta de formación pedagógica del profesorado.

Palabras claves: Estrategias metodológicas participativas, aprendizaje significativo.

Recibido: 9 de noviembre de 2016

Aceptado: 16 de diciembre de 2016

1 Doctorante en Educación e Intervención Social, UNAN-Managua, docente de FAREM-Estelí, UNAN-Managua. Correo electrónico: anatellezf@yahoo.com 


\title{
Methodological Strategies for the Meaningful Learning of Chemistry: A Study Conducted at FAREM-Estelí, UNAN-Managua, 2016
}

\begin{abstract}
The present article summarizes the main results of a study that dealt with methodological strategies that contribute to a meaningful learning of Chemistry, in two programs at FAREM-Estelí, UNANManagua, which were referred to as Program \#1 and Program \#2. The study found that the development of the learning process in one of the groups was appropriate and that there were difficulties in the other, as they were organizational and methodological inconveniences. It was proven that active methodological strategies contribute to a meaningful learning and motivation for the study of Chemistry. To that end, a compendium of participatory methodological strategies was designed. Those strategies were adapted to the context of our Faculty as a supporting resource to Chemistry teachers and our educational processes. The main research results confirm our research intuitions that, the implementation of non-active strategies limits a meaningful learning of Chemistry, which is related to the lack of teacher training.
\end{abstract}

Keywords: Participatory methodological strategies, meaningful learning. 


\section{INTRODUCCIÓN}

La UNAN-Managua plantea, en la fundamentación pedagógica del Modelo Educativo 2011, que el proceso de enseñanza-aprendizaje debe estar centrado en el estudiante. De ahí que, se pretende que éste asuma un rol activo, participativo y con una alta responsabilidad en el desarrollo de un aprendizaje autónomo y estratégico. Es en esta visión que se ha establecido la implementación de metodologías de aprendizaje activas.

En la Facultad Regional Multidisciplinaria de Estelí (FAREM-Estelí), una buena parte de los docentes estamos implementando estrategias metodológicas activas en el proceso de enseñanza aprendizaje. Sin embargo, hemos constatado dificultades de aprendizaje en disciplinas de ciencias, entre ellas la asignatura Introducción a la Química.

En las evaluaciones realizadas en los colectivos docentes y en las asambleas estudiantiles de las carreras del Departamento de Ciencia, Tecnología y Salud de nuestra Facultad, los docentes consideraron, entre otros aspectos, que los bajos rendimientos están asociados a la falta de interés, falta de motivación y poca identidad con el perfil profesional. También, que esto tiene que ver, con concepciones erróneas en relación a que son materias difíciles y aburridas.

Así mismo, los docentes son de la opinión que tiene que ver con la base que traen los estudiantes de la secundaria, falta de estudio individual, de escucha, de materiales y reactivos para la realización de más prácticas de laboratorio, que algunos estudiantes no aprovechan el tiempo y tienen dificultad en el análisis de problemas. Ello, ha venido siendo un tema de grandes preocupaciones de parte del profesorado de nuestra Facultad.

Referente a la asignatura Introducción a la Química, los estudiantes expresaron como principal dificultad, la poca relación que tiene los contenidos de la Química con la realidad y la limitada posibilidad de aplicación a la solución de problemas prácticos. Por tal razón, nos motivamos a indagar sobre ¿Qué efecto tienen las estrategias metodológicas en el proceso de aprendizaje de la asignatura Introducción a la Química? Así que, se consideró importante centrar este estudio en las "Estrategias metodológicas que favorecen un aprendizaje significativo en la asignatura Introducción a la Química en el contexto de nuestra Facultad, durante el segundo semestre de 2015".

En fin, la presente investigación resultó de gran interés, puesto que hizo una valoración de las estrategias metodológicas implementadas en la asignatura Introducción a la Química en dos carreras de nuestra Facultad. Además, se diseñó un compendio de metodologías participativas que contribuyan a la generación de aprendizajes significativos en dicha asignatura.

\section{Objetivos de investigación}

\section{Objetivo General}

- Determinar las estrategias metodológicas que favorecen un aprendizaje significativo en la asignatura Introducción a la Química en el contexto de la FAREM Estelí.

\section{Objetivos Específicos}

- Describir la conducción del proceso de aprendizaje de la asignatura Introducción a la Química.

- Fundamentar las estrategias metodológicas que propician el aprendizaje significativo en la asignatura Introducción a la Química.

- Disponeruncompendiodeestrategiasmetodológicas participativas acorde a un aprendizaje significativo en la asignatura Introducción a la Química.

\section{Hipótesis o "intuiciones" de investigación}

- En función de los resultados de este estudio, la utilización de estrategias y técnicas de enseñanza 
aprendizaje no activas en la asignatura introducción a la Química, limitan el aprendizaje significativo del estudiantado.

- La no utilización de estrategias y técnicas activas de enseñanza aprendizaje se debe a la falta de formación docente del profesorado.

\section{FUNDAMENTACIÓN TEÓRICA DE LA INVESTIGACIÓN}

Los planes de estudios y el modelo educativo de la Universidad Nacional Autónoma de Nicaragua, Managua (UNAN-Managua) tienen su fundamentación legal en el artículo 117 de la constitución política nicaragüense, el cual se lee:

La educación es un proceso único, democrático, creativo y participativo que vincula la teoría con la práctica, el trabajo manual con el intelectual y promueve la investigación científica. Se fundamenta en nuestros valores nacionales, en el conocimiento de nuestra historia, de la realidad, de la cultura nacional y universal y en el desarrollo constante de la ciencia y de la técnica; cultiva los valores propios del nuevo nicaragüense, de acuerdo con los principios establecidos en la presente Constitución, cuyo estudio deberá ser promovido.

En este sentido, en el Modelo Educativo de nuestra universidad se definen estrategias de aprendizaje a fin de preparar al estudiante (de manera individual y colaborativa), para ser un generador activo de conocimiento. Todo ello, con miras a la formación de técnicos y profesionales pensantes, críticos, creativos e innovadores, con sólidos valores y con un espíritu humanista; quizá este sea uno de los más grandes desafíos. (UNAN, 2011).

Es importante destacar, que el Modelo Educativo que impulsa nuestra alma mater está fundamentado en las políticas educativas del Estado, que expresan claramente el tipo de profesionales que demanda nuestra sociedad con miras a un desarrollo sostenible y sustentable.
En la teoría del aprendizaje significativo de David Ausubel, citado por Moreira (2010), se plantea, que se debe entender que el aprendizaje significativo es un aprendizaje con significado, comprensión, y con capacidad de aplicación a la práctica.

Moreira (2010), afirma que el aprendizaje significativo es el esperado por el docente como resultado de su acción educativa, pero que la práctica es muy distinta. Debido a que, entran en juego una serie de factores como son: la motivación e interés del estudiante, las estrategias metodológicas, experiencia y creatividad del docente y las condiciones ambientales; aspectos que deben ser considerados en la organización y planeación educativa.

De acuerdo con, Ballesta, Izquierdo \& Romero (2011):

El aprendizaje es significativo cuando el alumno relaciona los conocimientos a aprender y les da un sentido a partir de la estructura conceptual que ya posee, de su propia experiencia. El aprendizaje no es independiente del contexto en el que se realiza,... es activo cuando existe en el que aprende implicación, participación y protagonismo. La autoestima y la motivación aumentan enormemente las posibilidades formativas. Por tanto, el profesor debe intentar motivar a sus alumnos ayudándolos a descubrir sus necesidades $\mathrm{y}$ prioridades educativas. El valor educativo de la experiencia es indiscutible..., ya que la implicación personal se convierte en un fuerte estímulo motivador (p. 358).

Por lo antes expuesto, se puede afirmar, que el aprendizaje significativo se concretiza a partir de la organización y relacionalidad de contenidos, sin tomarlos al pie de la letra, ni realizando una mera transferencia. De modo que, el aprendizaje se da cuando hay congruencia entre lo que se facilita, se aprende, se contextualiza y se aplica. El hecho de que los estudiantes partan de sus propias experiencias y de lo que significa su realidad, les permite construir sus aprendizajes para la vida y por la vida. De manera que, los conocimientos se tornan interesantes y pertinentes; acorde con sus motivaciones y emociones. 
Lo que se pretende alcanzar en el aprendizaje significativo es aprender a aprender. Así pues, es de suma importancia en el proceso de enseñanza aprendizaje, "el desarrollo de estrategias cognitivas de exploración y de descubrimiento, de elaboración y de organización de la información, así como el proceso interno de planificación, regulación y evaluación de la actividad". (Vivas, N. 2010).

Paulo Freire, considera que el aprendizaje humano es siempre una construcción interior y reconoce el aprendizaje del "educador y del educando" en el proceso de enseñanza aprendizaje. Además, plantea que "el educador tiene como prioridad ayudar a la persona a lograr un punto de vista cada vez más crítico de su realidad, con la alta responsabilidad que este acto requiere”. Así que, nuestro papel como educadores es crear las oportunidades para que los estudiantes participen en la construcción y aporten "su verdad". Si marginamos a alguien corremos el riesgo de marginar la verdad o una parte importante de ella.

Matute, López \& Anzola (2011, p.50), consideran que "la enseñanza de la Química debe contribuir a la formación de estudiantes, que sean capaces de analizar y comprender las diferentes transformaciones dadas en el mundo que les rodea". Así que, la utilización de dichas estrategias metodológicas activas es fundamental para el logro de tan importante propósito. Con ello, se pretende la superación de las prácticas pedagógicas mecanicistas que, en vez de motivar al educando hacia un aprendizaje efectivo, más bien lo desmotivan al no poder visualizar la aplicabilidad del conocimiento a su práctica cotidiana.

En este mismo orden, Sandoval, Mandolesi \& Cura (2013), citando a Del Puy Pérez Echeverría et al., 1994, plantean que:

La química es una ciencia teórico-experimental calificada para movilizar la actividad cognitiva de los alumnos de forma creativa. De hecho, en un experimento de laboratorio se incorporan los órganos de la visión, audición, olfato y tacto aptos para ayudar a contemplar de manera conjunta el "¿cómo?”, el “¿por qué?” y el “¿para qué?” de lo que se aprende. (p. 129).

Así que, debemos potenciar la pedagogía de la pregunta y de la investigación como método de aprendizaje, en pro de la comprensión de los fenómenos químicos, intensificando el diálogo, recreando las relaciones, resignificando los contenidos el objeto de estudio como sujetos activos de su propio aprendizaje. Es importante, que en el proceso de aprendizaje de la Química se desarrolle en los estudiantes la capacidad de resolver problemas del entorno, de asignar significados a lo que se aprende, de aprender a adaptarse a nuevas situaciones y de sentirse implicados, como sujetos activos, en la transformación de la realidad.

Tomando en consideración, que las estrategias son las acciones que se planifican para lograr un fin o misión; conviene precisar, entonces, que las estrategias metodológicas activas son herramientas o acciones que el docente utiliza en el proceso de enseñanza aprendizaje. De igual manera, se afirma que éstas son una guía ordenada de las acciones educativas y permiten la identificación de procedimientos para la actuación del docente; en relación con la programación, implementación y evaluación del proceso de enseñanza aprendizaje.

Al respecto, López, F. (2007) plantea que en “educación, son importantes los contenidos, pero también la forma de impartirlos, es decir, la metodología que empleemos” (p. 358). Díaz \& Hernández (2001), citados por Matute, López \& Anzola (2011), definen las estrategias de enseñanza aprendizaje como "el conjunto de acciones graduales y sistemáticas que conforman todo el proceso de enseñanza y aprendizaje realizado por los docentes, con la finalidad de guiar al estudiante hasta la consolidación del conocimiento" (p.50).

Un aspecto relevante a destacar, es que las metodologías activas, o participativas, se ubican dentro de la pedagogía 
crítica, autogestionaria y humanística. En este sentido, López, F. (2007) define la metodología participativa como el "conjunto de procesos, procedimientos, técnicas y herramientas que implican activamente al alumno en el proceso de enseñanza/aprendizaje" ( $p$. 93). También, afirma que este enfoque metodológico interactivo se fundamenta en una "comunicación dialógica entre profesor alumno y alumno-alumno que potencia la implicación responsable del estudiante y que conlleva a la satisfacción y el enriquecimiento, tanto del docente como del alumno". (p. 94).

De igual manera, el autor destaca que la metodología participativa se basa en procesos activos de intercambio, es decir, de conocimientos, experiencias, vivencias, sentimientos, entre otros; y en la construcción colectiva de conocimientos que se propicia entre los sujetos que conforman el grupo. Considera que la educación, incluida la enseñanza superior, debe cederle protagonismo al estudiante y ser problematizadora, promoviendo el desarrollo de procesos críticos de enseñanza aprendizaje.

Conviene aclarar aquí, que el término metodología participativa en la literatura pedagógica tiene diversas denominaciones como: estrategias de enseñanza, estrategias de enseñanza-aprendizaje, estrategias o técnicas didácticas, estrategias docentes, entre otras. Sin embargo, éstas se utilizan indistintamente, puesto que sus fronteras son difusas y en ocasiones pueden ser utilizadas con múltiples significaciones; no obstante, sus ventajas son indudables, siempre y cuando su utilización sea pertinente en los procesos educativos.

Ahora bien, a fin de contribuir en los procesos de innovación y de cambio en la docencia universitaria, se describen en este apartado algunas metodologías participativas, entre las que destacan:

El Aprendizaje Basado en Problemas (ABP). Es "un método de aprendizaje basado en el principio de usar problemas como punto de partida para la adquisición e integración de los nuevos conocimientos" (Barrows, citado por Universidad Politécnica de Madrid, 2008, p.4). es una metodología centrada en el aprendizaje, orientada al análisis, la reflexión y la resolución de problemas

Aprendizaje Basado en Investigación (ABI). Tiene como propósito conectar la investigación con los procesos de aprendizaje, las cuales permiten la incorporación parcial o total del estudiantado en una investigación basada en métodos científicos.

Aprendizaje Orientado en Proyectos (AOP). Como metodología integradora consiste en la realización de un proyecto de algo concreto (real o una simulación cercana a la realidad), por parte del educando con la intención de solucionar una situación problemática que requiera soluciones prácticas. En este sentido, "se caracteriza por aplicar de manera práctica una propuesta que permite solucionar un problema desde diversas áreas de conocimiento, centrada en actividades y productos de utilidad social". (Pimienta, 2012, p.137).

Estudio de casos. Describe un suceso real o simulado complejo, para que los estudiantes apliquen sus conocimientos y habilidades y encuentren soluciones al mismo.

A continuación, se describen algunas técnicas participativas que nos comparte López (2007) y otros autores consultados.

Pequeño grupo de discusión: desarrolla la capacidad de reflexión y potencia la escucha entre los participantes. Se aprovechan los conocimientos, la experiencia de los estudiantes y facilita la comprensión de la realidad en forma de debate.

Aprendizaje cooperativo: fomenta la resolución creativa de problemas. Desarrolla el pensamiento creativo, valorando la actitud de creación y el aporte personal. Favorece la comunicación y el trabajo en equipo. 
Quinteto en rotación A-B-C-D-E: con la aplicación de esta técnica se contribuye al desarrollo de la capacidad de síntesis y reconstrucción de lo analizado, se facilita el diálogo y la escucha activa. Así mismo, se practica la adaptación a nuevos grupos y la asertividad. Para tal efecto, se divide a los estudiantes en grupos de cinco, para que debatan sobre un tema durante 10 minutos. Después, uno de los miembros del quinteto pasa a otro grupo, mientras se recibe a un miembro de un grupo diferente, el que llega actúa de relator del grupo de procedencia y continúan debatiendo sobre la temática. A los 10 minutos se procede a una nueva rotación y así sucesivamente, se podría concluir o no con un plenario.

Torbellino o lluvia de ideas: promueve la participación activa, se potencia la imaginación y creatividad, se promueve la libre expresión de ideas, por lo que debe evitarse cualquier manifestación que la limite.

Juego de roles: Se basa en el comprobado efecto que ejerce la actividad lúdica sobre el aprendizaje. Es así que, a través del juego las personas adquieren, modifican y desarrollan determinadas actitudes y habilidades con mayor facilidad.

Video-foros: consiste en el desarrollo de un contenido mediante la presentación de una película, video o documental. Se utiliza para introducir el debate sobre un tema de interés, favoreciendo la reflexión, el análisis crítico y el diálogo en el debate colectivo.

Preguntas didácticas: son cuestionamientos que desarrollan la comprensión en diversos campos del saber y constituyen una importante herramienta, dado que facilitan el desarrollo del pensamiento crítico, creativo y lógico.

Qué sé, qué quiero, qué aprendí (SQA). Ogle, (1986), citado por Pimienta, P. (2012), plantea que esta técnica permite motivar el estudio, indagando en los conocimientos previos, para después interrogar en lo que se desea aprender, y finalmente, comprobar lo que se ha aprendido.

En este mismo orden, resulta innegable la utilidad de las Tecnologías de la Información y la Comunicación adecuadamente empleadas como herramientas educacionales en el proceso de enseñanza aprendizaje de la Química. Este recurso didáctico motivaría a los estudiantes conforme van viviendo y experimentando esas nuevas experiencias de aprendizaje. Entre éstas se pueden utilizar los laboratorios virtuales, los blog y los juegos de simulación en diversos temas de Química.

\section{DISEÑO DE LA INVESTIGACIÓN}

El alcance de esta investigación, de acuerdo con sus objetivos y el nivel de profundidad, es de tipo descriptivo, ya que comprende la interpretación y análisis de los hechos, vivencias, circunstancias y experiencias en el proceso de enseñanza aprendizaje de la asignatura Introducción a la Química.

Según el tiempo de realización esta investigación es transeccional o transversal descriptiva. Los diseños de investigación de este tipo "recolectan datos en un sólo momento, en un tiempo único. Su propósito es describir variables y analizar su incidencia e interrelación en un momento dado" (Hernández et al 2012, p.151). De modo que, en este estudio pretendemos indagar y hacer una descripción detallada de las actividades, vivencias, emociones, hechos, experiencias y situaciones vividas durante el proceso de enseñanza aprendizaje de dicha asignatura.

La metodología empleada es fundamentalmente cualitativa, aunque algunos aspectos que han sido abordados cuantitativamente, para el procesamiento de datos que requerían de este tratamiento.

La población de este estudio está constituida por docentes y estudiantes que imparten y cursan, respectivamente, la asignatura Introducción a la 
Química en las carreras del departamento de Ciencia, Tecnología y Salud de la FAREM-Estelí. La misma está conformada por 271 estudiantes y tres docentes.

La muestra la conforman dos docentes y 88 estudiantes (inicialmente eran 92, pero hubo deserción) de dos carreras del Departamento de Ciencia Tecnología y Salud, a las que por razones éticas y para asegurar el anonimato de los participantes, las hemos denominado carrera número uno y carrera número dos. La técnica de muestreo empleada fue no probabilística e intencional, ya que las personas que participaron como sujetos de investigación fueron seleccionadas de acuerdo a criterios y conveniencia de la investigadora. También, por la voluntariedad de los participantes que impartieron o cursaron, respectivamente, la asignatura Introducción a la Química en dos carreras del departamento.

Lo anterior se justifica con lo planteado por Hernández et al (2012. p. 392) en relación a que en las investigaciones cualitativas "el tamaño de la muestra no es importante desde una perspectiva probabilística, pues el interés del investigador no es generalizar los resultados de su estudio a una población más amplia".

Las técnicas que se utilizaron para la recolección de la información fueron las siguientes:

Análisis documental: esta técnica se concretó a través de la revisión del Modelo Educativo de nuestra Universidad, programa y plan didáctico de la asignatura Introducción a la Química. El propósito fue seleccionar, analizar, evaluar, sintetizar, interpretar y triangular la información contenida en los documentos analizados, a la luz de la problemática objeto de estudio.

Observación: se observaron 15 sesiones de clases alternas en cada una de las carreras en diferentes momentos, teniendo presente el inicio y el final del semestre. Ello, permitió la identificación de las estrategias metodológicas aplicadas, que resultan ser más efectivas para el aprendizaje de la asignatura Introducción a la Química.

Entrevista: estuvo dirigida a los dos docentes que imparten la asignatura en las carreras de la muestra y a los coordinadores de carrera y director de departamento participantes en el estudio, para indagar sobre las estrategias metodológicas utilizadas en el proceso de aprendizaje de dicha asignatura.

Grupo focal: fue dirigido a catorce (nueve en la carrera número uno y cinco en la carrera número dos) estudiantes seleccionados al azar, quienes expresaron sus opiniones sobre las estrategias metodológicas implementadas por el docente, con el propósito de analizar la aceptación e incidencia de éstas en el proceso de aprendizaje de la asignatura.

Encuesta: nos permitió obtener información sobre aspectos de la conducción del proceso de aprendizaje de la asignatura Introducción a la Química y de las estrategias metodológicas más efectivas para el aprendizaje de la misma. La información obtenida se utilizó para el análisis cuantitativo, a fin de identificar y conocer la magnitud del problema objeto de estudio.

El procesamiento y análisis de los datos obtenidos durante la etapa de campo nos permitió extraer información relevante, en relación a la problemática objeto de estudio. En palabras de Hernández et al (2012), en el análisis de datos cualitativos el proceso esencial consiste en que recibimos datos no estructurados y los estructuramos e interpretamos, con el fin de llegar a interpretaciones razonables; dándole sentido a la información obtenida y organizándola para poder explicarla y describir el fenómeno objeto de estudio.

En ese sentido, en este estudio se hicieron reflexiones, interpretaciones, análisis, comparaciones y comprobaciones. De modo que, la organización, discusión y análisis de los resultados nos permitió 
dar una explicación lógica a la problemática de la investigación. El análisis documental se concretó mediante la revisión de bibliografía, Modelo Educativo, programa de asignatura, plan didáctico y plan de clase. Todo ello, con el propósito de analizar, interpretar y triangular la información obtenida en el proceso de investigación.

Los datos de la entrevista. grupo focal y guía de observación (registro de las sesiones de clase de Química), se procesaron usando el método de reducción. Para ello se estableció una categorización de las respuestas de acuerdo a los objetivos. Estos datos se interpretaron dando sentido a las descripciones de cada categoría a través de tablas y matrices, a fin de arribar a las conclusiones e implicaciones (propuesta de mejora).

Ahora bien, el procedimiento que se siguió para analizar los datos obtenidos mediante el cuestionario, inició con la organización, clasificación y reducción de los datos. Los datos se procesaron utilizando el software estadístico SPSS, versión 22 y con el programa Microsoft EXCEL, se elaboraron los histogramas de barra, gráficos de pastel y tablas de frecuencia.
Los resultados cualitativos y cuantitativos, aunque pueden mostrar divergencias, son complementarios, lo que favorece el análisis. La interpretación de los resultados, se realizó triangulando la información obtenida en las entrevistas, grupo focal, análisis documental, encuesta y observaciones; contrastándolos con la teoría descrita en el marco teórico. Ello nos permitió arribar a las conclusiones del estudio.

\section{ANÁLISIS E INTERPRETACIÓN DE LOS RESULTADOS}

Los resultados del presente trabajo investigativo se obtuvieron a partir del procesamiento, análisis y comparación de la información.

\section{Carrera número uno}

En el análisis del plan didáctico de la asignatura Introducción la Química, encontramos que la docente reflejó sugerencias metodológicas contempladas en el Modelo Educativo y en el programa de asignatura e incorporó otras. Además, incluye objetivos y contenidos conceptuales, procedimentales y actitudinales; así como las formas de evaluación con las siguientes modalidades:

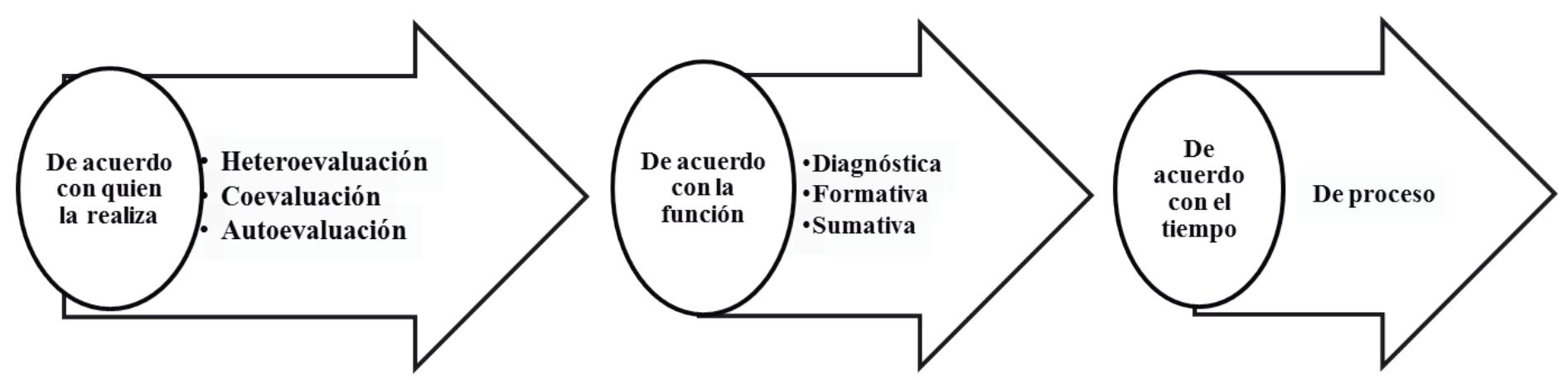


En cuanto a las estrategias metodológicas utilizadas en el proceso de aprendizaje de la Química, de los 37 estudiantes encuestados, el $60.7 \%$ mencionó la clase práctica, el $21.3 \%$ la clase expositiva y la menos mencionada fue la de los grupos de discusión con 1.6 $\%$. Los resultados en detalle, son presentados en el siguiente gráfico:

\section{Estrategias metodológicas utilizadas}

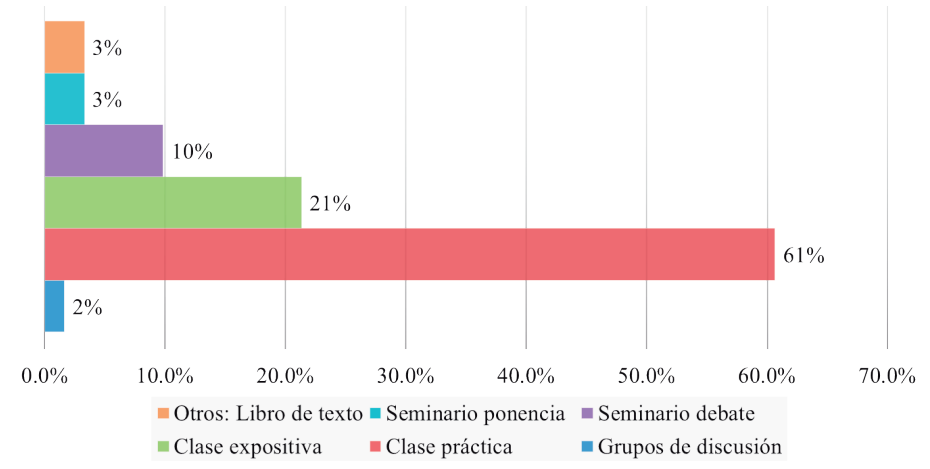

Estrategias metodológicas utilizadas en la carrera numero uno

Al respecto, los entrevistados fueron coincidentes al manifestar que en la carrera número uno se utilizaron estrategias metodológicas participativas. En este sentido, el director de departamento destacó, que las conferencias participativas permiten compartir información y debatir ideas, si éstas son bien planificadas.

De acuerdo con lo observado, la docente titular propiciaba la motivación en las clases que iba desarrollando a través de una serie de acciones y expresiones, por ejemplo, les comentaba acerca de la importancia del aprendizaje de la Química y lo fácil que puede resultar su comprensión. En las distintas sesiones, desarrolló los diferentes momentos didácticos. Las estrategias metodológicas participativas y los recursos didácticos utilizados fueron adecuados, acordes a cada uno de los momentos. En el marco de estas estrategias fue posible la recuperación de aprendizajes y experiencias previas.
Ahora bien, de acuerdo con lo observado y por lo expresado por los estudiantes en el grupo focal, la docente mantuvo buenas relaciones con el grupo clase y les brindaba confianza. Así mismo, existen otros factores que influyen en el grado de motivación que tienen los estudiantes, como el valor de utilidad para sus metas futuras. Así, por ejemplo, un estudiante expresó: "sentí inconformidad con la asignatura, pues no hay otras asignaturas que complementen a ésta, ¿para qué ver aspectos como los enlaces químicos, configuración electrónica, si no lo vamos a aplicar en nuestra carrera?".

En las sesiones de clase, promovió la evaluación de proceso, pues los contenidos se evaluaban conforme se iban desarrollando. Utilizó la evaluación diagnóstica, formativa, la autoevaluación y heteroevaluación. También, lo hacía de manera cualitativa, a través de preguntas orales como: ¿qué funcionó? ¿qué no funcionó? ¿qué debemos mejorar? Esto es relevante, ya que permite visualizar dificultades y alternativas de solución conjunta para la mejora continua.

\section{Carrera número dos}

En el plan didáctico de la asignatura de Química se visualiza que prevalece la conferencia como estrategia metodológica, matizada por la explicación del docente; predominando la estrategia metodológica tradicional, donde el docente es el cimiento y condición del acto educativo. Además, en éste se describen objetivos y contenidos conceptuales, procedimentales y actitudinales, pero los que prevalecen, de acuerdo con lo antes descrito, son los conceptuales. Como sabemos, la mayor parte de lo que se enseña se olvida y en general lo que recordamos, es irrelevante.

En ese sentido, de acuerdo con López, F. (2007):

La conferencia es la estrategia didáctica más pobre en cuanto a las competencias que desarrolla, ya que no impulsa competencias como el saber hacer, el trabajo en equipo, la comunicación, el liderazgo o la toma de decisiones, posicionándose en el mero saber. 
Ahora bien, la forma de evaluación consignada en plan didáctico de la asignatura Introducción a la Química hace referencia a las siguientes modalidades:
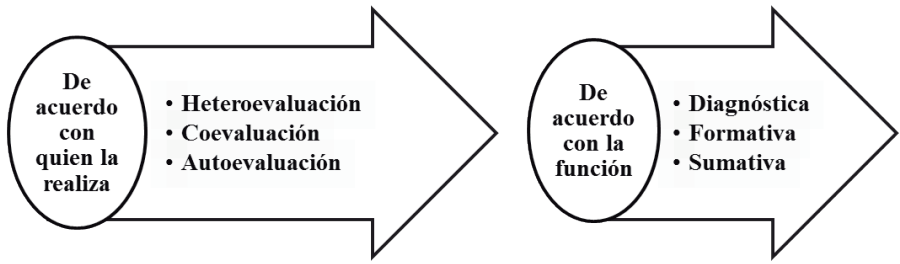

Referente a las estrategias metodológicas utilizadas por el docente, de los 30 estudiantes encuestados, el $53.8 \%$ mencionó la clase práctica; el $36.5 \%$, la clase expositiva y el $3.8 \%$, los grupos de discusión. Los resultados son mostrados en el siguiente gráfico:

\section{Estrategias metodológicas utilizadas}

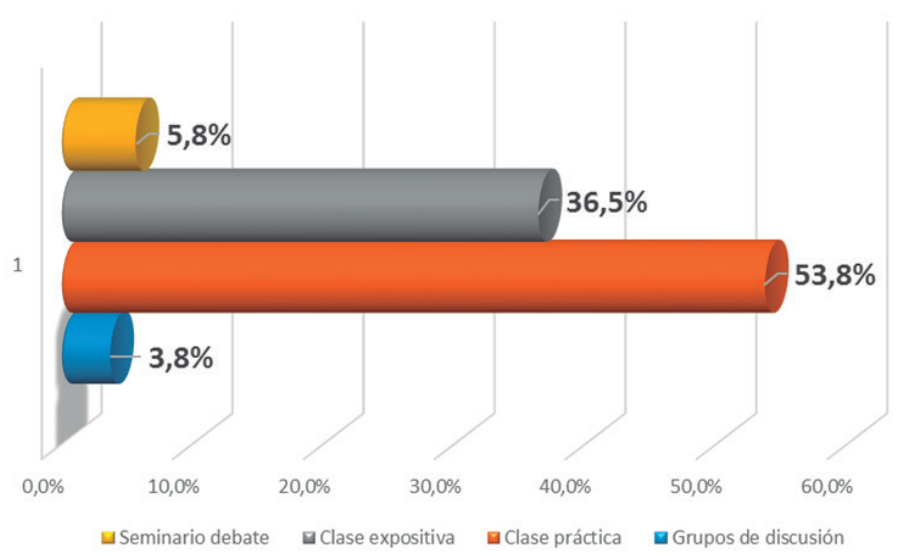

Estrategias metodológicas utilizadas en el proceso de aprendizaje en la carrera número dos.

Referente a la conducción del proceso de aprendizaje en las clases de Química, constatamos una marcada tendencia hacia la improvisación y la ausencia de planes diarios. Además, en ninguna de las sesiones se ambientó el salón de clases, de acuerdo con lo establecido en las metodologías participativas. Es importante destacar, que cuando las explicaciones se tornaban un tanto confusas, se escuchaban algunas expresiones de los estudiantes, como, por ejemplo, “¡ay señor Jesucristo, sálvame de este pecado”.

En general, el docente no guio a los estudiantes a situaciones de aprendizaje que los estimularan a cuestionar o a expresar sus dificultades con respecto a tal o cual aspecto de la clase, es decir, no se aplicó la pedagogía de la pregunta como estrategia idónea en el proceso. También, fueron notorias las dificultades de dominio sobre el grupo, lo que provocaba desorden. Así, por ejemplo, algunos estudiantes conversaban, otros silbaban y se salían de clase, aprovechando que el docente se quedaba por espacios prolongados leyendo en la computadora.

Se pudo notar, que mantenía una buena relación con algunos estudiantes, con los que siempre estaban atentos a la clase y que, por lo general, se aproximaban a él para aclarar dudas. Quizá, la no utilización de técnicas participativas hacía que algunos estudiantes se distrajeran y desorganizaran, provocando interferencias en la escucha de los que sí estaban interesados en las explicaciones. Así pues, encontramos muchos aspectos que ameritan su mejora. Además, en las sesiones de clase no se pudo verificar el cumplimiento total de los tres momentos didácticos, prevaleciendo la metodología tradicional. Ello, fue corroborado por los informantes y los sujetos de estudio.

La evaluación de la asignatura siguió lo establecido en la normativa de nuestra Universidad, para tal efecto. En opinión de los entrevistados y de acuerdo con lo observado, la evaluación del proceso de aprendizaje se realizó través de pruebas cortas, trabajos en equipo y un examen. Cabe señalar que, por orientaciones de la coordinación de carrera el docente asignó proyectos en cumplimiento a la inserción del eje transversal de innovación. Esto tuvo un efecto motivador, importante para el aprendizaje.

Los estudiantes de la carrera número uno, subrayaron que los trabajos grupales, las exposiciones (seminario ponencia), las investigaciones, las clases prácticas para la resolución de ejercicios, las ejemplificaciones y, las lecturas analíticas comprensivas en el libro de texto de Química, les ayudaron mucho en la comprensión de las temáticas. 
La mayoría de los estudiantes a pesar de que tuvieron cambio de docente con metodología y forma de evaluación diferente, dedicaron tiempo al estudio independiente; lo cual es parte fundamental del proceso de aprendizaje. Así mismo, esto es un indicador del interés, autonomía y responsabilidad mostrada.

Es así que, en la carrera número uno se utilizó una serie de estrategias metodológicas participativas que contribuyeron a favorecer aprendizajes significativos. Por ejemplo, lecturas analíticas y comprensivas, video foro, lluvia de ideas y las preguntas didácticas, mismas éstas, que tal como lo expresa Freire, citado por Zuleta (2005), ayudan a activar el pensamiento, a iniciar procesos de interacción, a encontrar solución a los problemas, plantearse nuevos problemas y nuevas situaciones de aprendizaje.

Los estudiantes de ambas carreras destacaron que la realización de las investigaciones en la clase de Química les permitió aprender más, pues las temáticas asignadas para su exposición eran interesantes. En este sentido, hubo quién hizo énfasis en que: "cuando exponíamos, estudiaba más y le entendía un poquito más".

De ahí que, las estrategias metodológicas interactivas, así como las de trabajo independiente o autónomo, tienen un efecto motivador en los estudiantes, predisponiéndolos a un aprendizaje cada vez más consciente, con miras a un aprendizaje con sentido, es decir, un aprendizaje significativo. Así pues, en consonancia con Van de Velde, H. (2016), cada persona es autora en el proceso de construcción de significados y por ende de su conciencia.

De acuerdo con lo expresado por los estudiantes encuestados, el 34\%, en ambas carreras, destacan el video foro sobre las otras estrategias metodológicas señaladas, como la que más les motivó hacia el aprendizaje de la asignatura. Así mismo, en las dos carreras de la muestra, un $30 \%$ dijo que con preguntas.
En la carrera número uno, indicaron en un $13 \%$ que con reflexiones y en un $11 \%$ mediante dinámicas, tal como podemos apreciar en los gráficos siguientes:

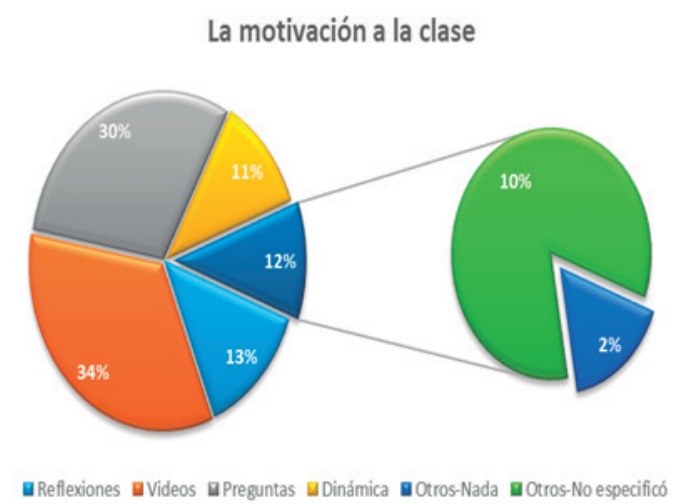

Carrera número uno

La motivación a la clase

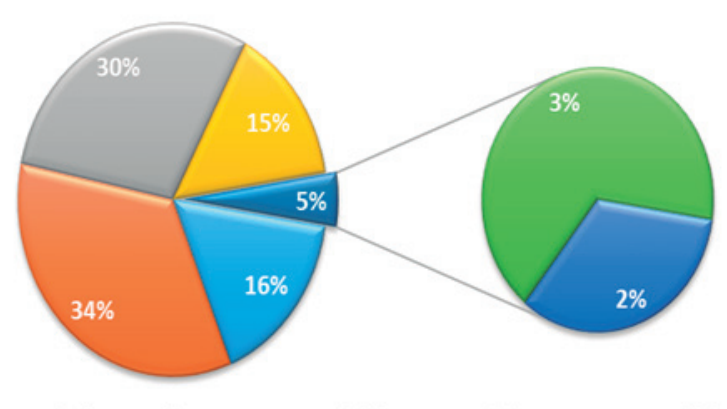

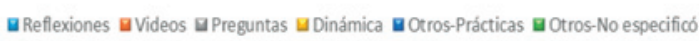

Carrera número dos

El $16 \%$ de los estudiantes de la carrera número dos, mencionó que la motivación en la clase de Química se genera a través de reflexiones y solamente un $2 \%$ que, mediante prácticas.

Tomando en cuenta los datos anteriores, podemos decir que, el proceso de aprendizaje depende de la habilidad del docente para mantener y mejorar la motivación de los estudiantes hacia el aprendizaje significativo de la Química

Referente a las estrategias metodológicas que propician aprendizajes significativos, las autoridades, en ambas carreras, coincidieron en manifestar que las prácticas de laboratorio son una de las estrategias más apropiadas para el aprendizaje significativo de la asignatura Introducción a la Química. Esto es coincidente con 
lo expresado por los estudiantes de la carrera número dos participantes del grupo focal, quienes al referirse a sus proyectos destacaron los aprendizajes obtenidos a través de las actividades desarrolladas.

Así que, las condiciones ambientales, la motivación intrínseca y extrínseca, la experiencia, la formación pedagógica y la creatividad, son aspectos fundamentales en la conducción o facilitación del proceso de aprendizaje. Todo ello, con miras a "transformar y mejorar la vida colectiva que se da en clase y dando respuestas a las necesidades e intereses del aula", (López, F., 2007, p. 132).

Como aportación práctica para el profesorado de Química y resultado principal de este estudio, se realizó una recopilación de propuestas de diversos autores sobre estrategias metodológicas participativas, las que han sido adaptadas y modificadas al contexto de nuestra Facultad. Ello, con el propósito de contribuir a generar procesos de aprendizajes significativos en la asignatura Introducción a la Química. Pues, tal como lo demuestran los resultados de la investigación, no es pertinente que los docentes de Química sigamos desarrollando nuestra labor docente con métodos pedagógicos tradicionales, que sólo sirven para potenciar la transmisión mecánica de conocimientos o de saberes sin sentido.

Es importante subrayar que, el compendio brinda estrategias metodológicas que invitan al análisis y reflexión, a la argumentación y experimentación, a la resolución de problemas, a la creatividad e innovación $\mathrm{y}$ al disfrute que provoca el aprender haciendo. También, se incluye el juego entre las técnicas participativas para hacer el aprendizaje más vivencial y motivador. Todo ello, dirigido a la búsqueda constante de conocimientos y aprendizajes significativos en la asignatura Introducción a la Química.

\section{CONCLUSIONES}

Como conclusión general del presente estudio, en la carrera número uno, consideramos particularmente satisfactorio que en el proceso de aprendizaje de la asignatura Introducción a la Química, bajo la facilitación de la docente titular, se implementaron estrategias metodológicas participativas. Las mismas contribuyeron a favorecer un aprendizaje con sentido, es decir, un aprendizaje significativo, según refieren los sujetos de estudio y los informantes clave.

Sin embargo, a juzgar por las valoraciones de los estudiantes, de la coordinación de la carrera y de las observaciones realizadas, el profesor interino utilizó una metodología tradicional. Es por esta razón, que los sujetos de estudio e informantes clave, sugieren que sea más dinámico, empático, que dé confianza y sea imparcial.

En la carrera número dos prevaleció la metodología tradicional, centrada en la transmisión de conocimientos. Es así que, el protagonismo residió en la figura del docente y en la transmisión mecánica de los mismos, conduciendo a la desmotivación en aquellos estudiantes poco aplicados e inquietos.

Como conclusiones más específicas, podemos distinguir algunas situaciones que valoramos positivamente. Referente al primer objetivo específico relacionado con la conducción metodológica, en la carrera número uno, el proceso de aprendizaje de la asignatura Introducción a la Química, fue conducido, dirigido y planificado de forma organizada; atendiendo a una secuencia lógica de acuerdo con los momentos didácticos que sugieren las ciencias pedagógicas. Ello, contribuyó a que el proceso de aprendizaje se desarrollara en un clima de confianza, favoreciendo de esta manera la interacción entre la docente y los estudiantes, entre sí y con los medios didácticos. 
En cambio, en la carrera número dos, la conducción del proceso de aprendizaje presentó algunos inconvenientes de orden metodológico y organizativo; lo que está relacionado con un déficit en la formación pedagógica y en la planificación docente. En raras ocasiones observamos en las sesiones de clase una secuencia lógica, en relación con los tres momentos didácticos propuestos por las ciencias pedagógicas, en aras de una comunicación y facilitación didáctica eficaz.

No obstante, el docente de la carrera número dos, dirigió proyectos durante el transcurso de la asignatura. En los mismos, los estudiantes tuvieron la oportunidad de elaborar productos alimenticios. Esto tuvo un impacto positivo, ya que se promovió el aprender haciendo y se vinculó, en alguna medida, la teoría con la práctica. Otro elemento motivacional, es que dichos proyectos fueron seleccionados para presentarse en la Jornada Universitaria de Desarrollo Científico de la Facultad.

Ahora bien, se constató, en ambas carreras, que las estrategias metodológicas activas son las que propician aprendizajes significativos en Química. Ello, se evidenció en las expresiones, tanto verbales como gestuales de los estudiantes. Por ejemplo, en la carrera número uno, destacaron las relaciones de comunicación que fomentaba la docente y la empatía surgida de la convivencia. Esto, se explica con la participación activa de los estudiantes, generada en la interacción grupal y en la discusión en plenaria. Así, el estilo de enseñanza aprendizaje flexible y activo, evidencia la maestría pedagógica de la docente.

En fin, las estrategias metodológicas más enriquecedoras para el aprendizaje significativo de la Química y que favorecieron aprendizajes activos, interactivos, colaborativos y autónomos, así como el aprender haciendo; fueron las estrategias metodológicas participativas. Entre las que destacan, la pedagogía de la pregunta, contextualización de los contenidos, lectura analítica comprensiva (haciendo uso del texto base, por ejemplo: resolución de ejercicios y problemas, estudios de caso, esquemas y juegos didácticos), video foro, lluvia de ideas, aprendizaje colaborativo, seminario ponencia, grupos de discusión y aprendizaje por proyectos.

Referente a la disposición de un compendio de estrategias metodológicas participativas, con miras a la significación de los aprendizajes en la asignatura de Química, se realizó una recopilación de metodologías y técnicas activas. Éstas fueron adaptadas a la asignatura y al contexto de nuestra Facultad. El mismo, servirá de apoyo a los docentes de Química, en función de su planificación didáctica $\mathrm{y}$, por ende, para la promoción de aprendizajes significativos en el estudio de esta asignatura. Así mismo, pretende contribuir a la solución de dificultades metodológicas identificadas en esta investigación.

Queremos destacar que en nuestro estudio se han confirmado las hipótesis o "intuiciones" investigativas. Así pues, en nuestros hallazgos constatamos que la utilización de estrategias y técnicas de aprendizaje no activas en la asignatura introducción a la Química, limitó el aprendizaje significativo de los estudiantes. También, se corroboró que la no utilización de estrategias y técnicas activas de enseñanza aprendizaje, se debe a déficit en la formación pedagógica de uno de los docentes participantes en el estudio.

En ese sentido, se sugiere a los docentes del área de Química de nuestra Facultad, adaptar las estrategias metodológicas propuestas acorde con la realidad que se vive en los salones de clase. A los que adolecen de una sólida formación pedagógica, prepararse continuamente en aras de ir mejorando su práctica pedagógica. Esto en función de ir fortaleciendo la comunicación didáctica, en pro de un aprendizaje con sentido, concediendo al estudiante un mayor protagonismo. 


\section{IMPLICACIONES}

Uno de los réditos más importantes de este trabajo investigativo, es disponer de un compendio de estrategias y técnicas metodológicas participativas, a fin de estimular el interés del estudiantado por el aprendizaje de la asignatura Introducción a la Química. De ello, deriva la importancia de enfatizar, que la interacción educador- educando, en el proceso de aprendizaje debe ser activa, participativa, reflexiva, analítica y creativa, como presupuesto básico para la construcción y apropiación consciente de los conocimientos.

A las autoridades de nuestra Facultad, se les sugiere realizar capacitaciones en temas relacionados a las metodologías y técnicas participativas para la mejora continua del proceso de aprendizaje de la Química, pues hemos intercambiado experiencias sobre metodologías activas, pero no específicamente en el área de esta disciplina. En aras de hacer efectiva la retroalimentación del proceso de capacitación propuesto, es pertinente el aseguramiento de un proceso de evaluación y autoevaluación sistemático y participativo, que sea aplicable tanto por los docentes de Química, como por las coordinaciones de carrera y la dirección del departamento de Ciencia, Tecnología y Salud.

Como futuras líneas de investigación, proponemos trabajar con laboratorios virtuales, dirigidas a la simulación de ensayos químicos en entornos virtuales de aprendizaje. Ello con el propósito de incrementar la motivación de los estudiantes hacia entornos tecnologizados y por la habilidad que inicialmente tienen en el manejo de simuladores e instrumentos informáticos.

\section{REFERENCIAS BIBLIOGRÁFICAS}

Ballesta, F., Izquierdo, T. \& Romero, B. (2011). Percepción del alumnado de Pedagogía ante el uso de metodologías activas. Educatio Siglo XXI, v. 29, n.2. Recuperado de http://goo.gl/X1T0Bf

Hernández, R., Fernández, C. \& Baptista, M. (2012). Metodología de la Investigación. México: McGRAW-HILL.

López, F. (2007). Metodología Participativa en la Enseñanza Universitaria. Madrid: NARCEA, S.A.

Matute, S., López, B. \& Anzola, Y. (2011). Estrategias de enseñanza basadas en el estudiante a partir del aprendizaje de las reacciones químicas. Educación y Humanismo. v. 13, n. 20. Recuperado de http:// goo.g1/3Ee4Z9

Moreira, M. (2010) ¿Por Qué Conceptos? ¿Por Qué Aprendizaje Significativo? ¿Por Qué Actividades Colaborativas? y ¿Por Qué Mapas Conceptuales? Revista QURRICULUM. n. 21 Recuperado de http://goo.gl/PH5NhR

Pimienta, J. (2012). Estrategias de enseñanzaaprendizaje. Docencia universitaria basada en competencias. México: PEARSON EDUCACIÓN.

Sandoval, M. J, Mandolesi, M.E \& Cura, R. (2013). Estrategias didácticas para la enseñanza de la química en la educación superior. Educación y Educadores. v. 16, n. 1. Recuperado de www. redalyc.org/pdf/834/83428614007.pdf

UNAN-Managua. (2011). Modelo Educativo, Normativa y Metodología para la Planificación Curricular. Managua: Editorial Universitaria.

UNAN-Managua. (2011). Plan Estratégico Institucional 2011-2015. Managua: Editorial Universitaria.

Universidad Politécnica de Madrid.(2008). Aprendizaje Basado en Problemas. Guías rápidas sobre nuevas metodologías. Madrid: Servicio de Innovación Educativa de la (UPM). Recuperado de http://goo. $\mathrm{gl} / \mathrm{ca} 9 \mathrm{mwb}$

Van de Velde, H. (2016). Pedagogía de la significación. Estelí: ABACOenRed. 